\title{
Fixed Point Results in JS-Multiplicative Metric Spaces
}

\author{
Abdullah Shoaib", Qaiser Mehmood \\ Department of Mathematics and Statistics, Riphah International University, Islamabad - 44000, Pakistan \\ *Corresponding author: abdullahshoaib15@yahoo.com
}

Received September 22, 2018; Revised November 11, 2018; Accepted December 06, 2018

\begin{abstract}
In this paper, we have introduced JS-multiplicative metric space and proved some fixed point theorems in this space. This new metric function is a generalized form of several functions such as multiplicative metric, dislocated multiplicative metric, multiplicative b-metric and multiplicative b-metric-like.
\end{abstract}

Keywords: fixed point, Js multiplicative metric space, multiplicative metric space, multiplicative b-metric-like space, multiplicative b-metric space

2010 Mathematics Subject Classification: 46S40; 47H10; 54H25.

Cite This Article: Abdullah Shoaib, and Qaiser Mehmood, "Fixed Point Results in JS-Multiplicative Metric Spaces.” Turkish Journal of Analysis and Number Theory, vol. 6, no. 6 (2018): 159-163. doi: 10.12691/tjant-6-6-3.

\section{Introduction}

Ozaksar and Cevical [1] investigated multiplicative metric space and proved its topological properties. Mongkolkeha et al. [2] described the concept of multiplicative proximal contraction mapping and proved best proximity point theorems for such mappings. Recently, Abbas et al. [3] proved some common fixed point results of quasi weak commutative mappings on a closed ball in the setting of multiplicative metric spaces. They also describe the main conditions for the existence of common solution of multiplicative boundary value problem. For further results on multiplicative metric space, see [4,5,6,7]. In 2017, Ali et al. [8] introduced the notion of $b$-multiplicative and proved some fixed point result. As an application, they established an existence theorem for the solution of a system of Fredholm multiplicative integral equations. Bakht Zada and Usman Riaz [9] introduced the idea of multiplicative b-metric-like space. Jleli and Samet [10] introduce a new generalization of metric space called generalized metric space (Js-metric space) and proved some fixed point theorems (see $[11,12,13]$ for further results).

In this paper, we present a new concept of Js multiplicative metric space that covers different spaces including multiplicative metric space, multiplicative b-metric space and multiplicative b-metric-like space. Also we prove Ciric type fixed point theorem and some fixed point theorems with partial order in Js multiplicative metric space.

\section{Js Multiplicative Metric Space}

Definition 2.1 Let $\mathcal{X} \neq \varphi$ and let $\Psi: \mathcal{X} \times \mathcal{X} \rightarrow[1,+\infty)$ be a mapping. For all $w \in \mathcal{X}$, we define the set $B(\Psi, \mathcal{X}, w)$ as follows:

$$
B(\Psi, \mathcal{X}, w)=\left\{\left\{w_{n}\right\} \subset \mathcal{X}: \lim _{n \rightarrow \infty} \Psi\left(w_{n}, w\right)=1\right\} .
$$

Definition 2.2 Let $\mathcal{X} \neq \varphi$ and $\Psi: \mathcal{X} \times \mathcal{X} \rightarrow[1,+\infty)$ be a given mapping. Then $(\mathcal{X}, \Psi)$ is called Js-multiplicative metric space, if it satisfies the following conditions:

$\left(\Psi_{1}\right)$ For all $w, y \in \mathcal{X}$, we have $\Psi(w, y)>1$ and $\Psi(w, y)=1 \Rightarrow w=y$;

$\left(\Psi_{2}\right)$ For all $w, y \in \mathcal{X}$, we have $\Psi(w, y)=\Psi(y, w)$;

$\left(\Psi_{3}\right)$ there exists $h>0$ such that if for all $(w, y) \in \mathcal{X} \times \mathcal{X},\left\{w_{n}\right\} \in B(\Psi, \mathcal{X}, w)$, then

$$
\Psi(w, y) \leq \lim \sup _{n \rightarrow \infty} \Psi\left(w_{n}, y\right)^{h} .
$$

The pair $(\mathcal{X}, \Psi)$ is called a Js multiplicative metric space. Remark 2.3 Clearly if the set $B(\Psi, \mathcal{X}, w)$ is empty for all $w \in \mathcal{X}$, then $(\mathcal{X}, \Psi)$ is a Js multiplicative metric space if and only if $\left(\Psi_{1}\right)$ and $\left(\Psi_{2}\right)$ are satisfied.

Example 2.4 Let $\mathcal{X}=[0,+\infty)$ and let $\Psi: \mathcal{X} \times \mathcal{X} \rightarrow[1, \infty)$ be define by

$$
\Psi(w, y)=\alpha^{(w+y)^{2}},
$$

where $\alpha>1$ be a finite fixed real number is a Js multiplicative metric space for all $w, y, \in \mathcal{X}$. Let $z_{n} \in B(\Psi, \mathcal{X}, w)$, then

$$
\lim _{n \rightarrow \infty} \Psi\left(z_{n}, w\right)=1 .
$$

So $z_{n}<\infty$ except possibly for finite number of terms. Let $p$ be the smallest natural number such that $z_{n}<\infty$, then 


$$
\begin{aligned}
\Psi(w, y) & =\alpha^{(w+y)^{2}} \leq \alpha^{\left(w+z_{n}+z_{n}+y\right)^{2}}, \text { for all } n \geq p \\
& =\alpha^{\left(w+z_{n}\right)^{2}+\left(z_{n}+y\right)^{2}+2\left(w+z_{n}\right)\left(z_{n}+y\right)} \\
& \leq \alpha^{2\left[\left(w+z_{n}\right)^{2}+\left(z_{n}+y\right)^{2}\right]} \\
& =\left[\alpha^{\left(w+z_{n}\right)^{2}} \cdot a^{\left(z_{n}+y\right)^{2}}\right]^{2}
\end{aligned}
$$$$
\Psi(w, y) \leq \lim \sup \Psi\left(z_{n}, y\right)^{2} \text {. }
$$

So $\left(\Psi_{3}\right)$ hold with $h=2$. Clearly, $\left(\Psi_{1}\right)$ and $\left(\Psi_{2}\right)$ hold. Thus $(\mathcal{X}, \Psi)$ is Js multiplicative metric space.

Definition 2.5 A sequence $\left\{w_{n}\right\}$ in Js multiplicative metric space is converges to $w \in \mathcal{X}$, if

$$
w_{n} \in B(\Psi, \mathcal{X}, w) .
$$

Proposition 2.6 Let $(\mathcal{X}, \Psi)$ be Js multiplicative metric space. Suppose $\left\{w_{n}\right\}$ is a sequence in $\mathcal{X}$ and $w, y \in \mathcal{X}$. If $\left\{w_{n}\right\}$ is convergent to both $w$ and $y$, then $w=y$.

Definition 2.7 A sequence $\left\{w_{n}\right\}$ in Js multiplicative metric space $(\mathcal{X}, \Psi)$ is called Cauchy, if

$$
\lim _{m, n \rightarrow \infty} \Psi\left(w_{n}, w_{n+m}\right)=1 .
$$

Definition 2.8 A Js multiplicative metric space $(\mathcal{X}, \Psi)$ is called complete if every Cauchy sequence in it is convergent to some element in $\mathcal{X}$.

Definition 2.9 Let $\mathcal{X} \neq \varphi$ and a function $\mathcal{B}: \mathcal{X} \times \mathcal{X} \rightarrow[1,+\infty) \quad$ is called a multiplicative b-metric-like on $\mathcal{X}$ if $\mathcal{B}$ satisfies the following conditions for all $w, y, z \in \mathcal{X}$ and a constant $s \geq 1$

$\left(\mathcal{B}_{1}\right) \mathcal{B}(w, y)=1$, then $w=y$;

$\left(\mathcal{B}_{2}\right) \quad \mathcal{B}(w, y)=\mathcal{B}(y, w)$;

$\left(\mathcal{B}_{3}\right) \mathcal{B}(w, z) \leq[\mathcal{B}(w, y) \cdot \mathcal{B}(y, z)]^{s}$.

The pair $(\mathcal{X}, \mathcal{B})$ is called a multiplicative b-metric-like space. If we take $s=1$, then $(\mathcal{X}, \mathcal{B})$ becomes dislocated multiplicative metric space. If we take $w=y$, then $\mathcal{B}(w, y)=1$, then $(\mathcal{X}, \mathcal{B})$ becomes multiplicative b-metric space. If we take $w=y$, then $\mathcal{B}(w, y)=1$ and $s=1$, then $(\mathcal{X}, \mathcal{B})$ becomes multiplicative metric space.

Proposition 2.10 Every multiplicative b-metric-like space, multiplicative b-metric space, dislocated multiplicative metric space and multiplicative metric space is Js multiplicative metric space.

Let $\mathcal{Y} \neq \varphi$ and let $J: \mathcal{Y} \times \mathcal{Y} \rightarrow[0,+\infty)$ be a given mapping. For all $x \in \mathcal{Y}$, let us define the set $B(J, \mathcal{Y}, x)$ as below

$$
B(J, \mathcal{Y}, x)=\left\{\left\{x_{n}\right\} \subset \mathcal{Y}: \lim _{n \rightarrow \infty} J\left(x_{n}, x\right)=0\right\} .
$$

Definition 2.11 [10]

Let $\mathcal{Y} \neq \varphi$ and $J: \mathcal{Y} \times \mathcal{Y} \rightarrow[0,+\infty)$ be a given mapping. Then $(\mathcal{Y}, J)$ is called Js-metric space if it satisfies the following conditions for all $x, y \in \mathcal{Y}$ :

$\left(J_{1}\right) J(x, y)=0 \Rightarrow x=y$;

$\left(J_{2}\right) \quad J(x, y)=J(y, x)$;

( $\left.J_{3}\right)$ there exists $h>0$ such that $\forall(x, y) \in \mathcal{Y} \times \mathcal{Y}$, and $\left\{x_{n}\right\} \in B(J, \mathcal{Y}, x)$

$$
J(x, y) \leq h \limsup _{n \rightarrow \infty} J\left(x_{n}, y\right) .
$$

Remark 2.12 Every Js metric space $(\mathcal{X}, d)$ generates a Js multiplicative metric space $(\mathcal{X}, \Psi)$ defined as

$$
\Psi(x, y)=e^{d(x, y)} .
$$

\section{Ciric Type Result in a Js multiplicative Metric Space}

This section deals with fixed point for Ciric type mappings in Js multiplicative metric space.

Definition 3.1 Let $(\mathcal{X}, \Psi)$ be a Js multiplicative metric space and $\mathcal{T}: \mathcal{X} \rightarrow \mathcal{X}$ be a self mapping. Let $k \in(0,1)$ then $\mathcal{T}$ is called Js k-quasicontraction if

$$
\Psi(\mathcal{T} w, T y) \leq[J(w, y)]^{k} \text { for all } w, y \in \mathcal{X},
$$

where

$$
J(w, y)=\max \left\{\begin{array}{l}
\Psi(w, y), \Psi(w, T(w)), \\
\Psi(y, T(y)), \Psi(w, T(y)), \\
\Psi(y, T(w))
\end{array}\right\} .
$$

Proposition 3.2 Let $(\mathcal{X}, \Psi)$ be a Js multiplicative metric space and $\mathcal{T}$ be a Js k-quasicontraction for some $k \in(0,1)$. Then any fixed point $u \in \mathcal{X}$ of $\mathcal{T}$ satisfies

$$
\Psi(u, u)<\infty \Rightarrow \Psi(u, u)=1 .
$$

Theorem 3.3 Let $(\mathcal{X}, \Psi)$ be complete Js multiplicative metric space and let $\mathcal{T}$ be a Js k-quasicontraction mapping for some $k \in(0,1)$ such that $h k<1$. If there exists an element $w_{0} \in \mathcal{X}$ such that $\eta\left(\Psi, \mathcal{T}, w_{0}\right)<\infty$ then the sequence $\left\{\mathcal{T}^{n}\left(w_{0}\right)\right\}$ converges to $u \in \mathcal{X}$ and if $\Psi\left(w_{0}, \mathcal{T} u\right)<\infty$ and $\Psi(u, \mathcal{T} u)<\infty$ then ' $u$ ' is the fixed point of $\mathcal{T}$. Moreover for each fixed point $u^{\prime} \in \mathcal{X}$ of $\mathcal{T}$ such that $\Psi\left(u, u^{\prime}\right)<\infty$ and $\Psi\left(u^{\prime}, u^{\prime}\right)<\infty$ then $u=u^{\prime}$.

Proof We shall prove that $\left\{\mathcal{T}^{q}\left(w_{0}\right)\right\}$ is a Cauchy sequence. Let $q \in \mathbb{N}_{0}(q \geq 1)$, as $\mathcal{T}$ is a Js kquasicontraction, for each $i, j \in \mathbb{N}_{0}$, we have 


$$
\begin{aligned}
& \Psi\left(\mathcal{T}^{q+i} w_{0}, \mathcal{T}^{q+j} w_{0}\right) \\
& \leq \max \left\{\begin{array}{l}
\Psi\left(\mathcal{T}^{q-1+i} w_{0}, \mathcal{T}^{q-1+j} w_{0}\right), \\
\Psi\left(\mathcal{T}^{q-1+i} w_{0}, \mathcal{T}^{q+i} w_{0}\right), \\
\Psi\left(\mathcal{T}^{q-1+i} w_{0}, \mathcal{T}^{q+j} w_{0}\right), \\
\Psi\left(\mathcal{T}^{q-1+j} w_{0}, \mathcal{T}^{q+j} w_{0}\right), \\
\Psi\left(\mathcal{T}^{q-1+j} w_{0}, \mathcal{T}^{q+i} w_{0}\right)
\end{array}\right\} .
\end{aligned}
$$

As

$$
\begin{aligned}
& \eta\left(\Psi, \mathcal{T}, \mathcal{T}^{q-1} w_{0}\right) \\
& =\sup \left\{\Psi\left(\mathcal{T}^{q-1+i}(w), \mathcal{T}^{q-1+j}(w)\right): i, j \in \mathbb{N}_{0}\right\} .
\end{aligned}
$$

So, (1) implies

$$
\begin{aligned}
\eta\left(\Psi, \mathcal{T}, \mathcal{T}^{q} w_{0}\right) & \leq \eta\left(\Psi, \mathcal{T}, \mathcal{T}^{q-1} w_{0}\right)^{k} \\
& \leq \eta\left(\Psi, \mathcal{T}, \mathcal{T}^{q-2} w_{0}\right)^{k^{2}} .
\end{aligned}
$$

So, we obtain

$$
\eta\left(\Psi, \mathcal{T}, \mathcal{T}^{q}\left(w_{0}\right)\right) \leq \eta\left(\Psi, \mathcal{T}, w_{0}\right)^{k^{q}} .
$$

Taking (2) into account and by the Definition of $\eta$, for every $m, q \in \mathbb{N}_{0}$, we have

$$
\begin{aligned}
\Psi\left(\mathcal{T}^{q} w_{0}, \mathcal{T}^{q+m} w_{0}\right) & \leq \eta\left(\Psi, \mathcal{T}, \mathcal{T}^{q} w_{0}\right) \\
& \leq \eta\left(\Psi, \mathcal{T}, w_{0}\right)^{k^{q}} .
\end{aligned}
$$

Using the fact that $k \in(0,1)$ and $\eta(\Psi, \mathcal{T}, w)<\infty$, we get

$$
\left.\lim _{q, m \rightarrow \infty} \Psi\left(\mathcal{T}^{q} w_{0}\right), \mathcal{T}^{q+m} w_{0}\right)=1
$$

This shows that $\mathcal{T}^{q}\left(w_{0}\right)$ is a Cauchy sequence. By completeness of $(\mathcal{X}, \Psi)$, we must have some $u \in \mathcal{X}$ such that $\mathcal{T}^{q}\left(w_{0}\right)$ is convergent to $u$. Now, we suppose that $\Psi\left(w_{0}, \mathcal{T} u\right)<\infty$. Using the inequality (2)

$$
\Psi\left(\mathcal{T}^{q} w_{0}, \mathcal{T}^{q+m} w_{0}\right) \leq \eta\left(\Psi, \mathcal{T}, w_{0}\right)^{k^{q}} .
$$

For every $q, m \in \mathbb{N}_{0}$, by the property $\left(\Psi_{3}\right)$, there exists some constant $h>0$ such that

$$
\begin{aligned}
& \Psi\left(u, \mathcal{T}^{q} w_{0}\right) \leq \lim \sup _{m \rightarrow \infty} \Psi\left(\mathcal{T}^{q} w_{0}, \mathcal{T}^{q+m} w_{0}\right)^{h} \\
& \leq \eta\left(\Psi, \mathcal{T}, w_{0}\right)^{h k^{q}} \text { for every } q \in \mathbb{N}_{0} .
\end{aligned}
$$

Now,

$\Psi\left(\mathcal{T}_{w_{0}}, \mathcal{T} u\right) \leq \max \left\{\begin{array}{l}\Psi\left(w_{0}, u\right), \Psi\left(w_{0}, \mathcal{T}_{w_{0}}\right), \Psi(u, \mathcal{T} u), \\ \Psi\left(\mathcal{T}_{w_{0}}, u\right), \Psi\left(w_{0}, \mathcal{T} u\right)\end{array}\right\}^{k}$

By using (3) and (4), we obtain

$$
\Psi\left(\mathcal{T w}_{0}, \mathcal{T} u\right) \leq \max \left\{\begin{array}{l}
\eta\left(\Psi, \mathcal{T}, w_{0}\right)^{h k}, \eta\left(\Psi, \mathcal{T}, w_{0}\right)^{k}, \\
\Psi(u, \mathcal{T} u)^{k}, \Psi\left(w_{0}, \mathcal{T} u\right)^{k}
\end{array}\right\} .
$$

Again, using the above inequality, we have

$$
\Psi\left(\mathcal{T}^{2} w_{0}, \mathcal{T} u\right) \leq \max \left\{\begin{array}{l}
\eta\left(\Psi, \mathcal{T}, w_{0}\right)^{h k^{2}}, \eta\left(\Psi, \mathcal{T}, w_{0}\right)^{k^{2}}, \\
\Psi(u, \mathcal{T} u)^{k}, \Psi\left(w_{0}, \mathcal{T} u\right)^{k^{2}}
\end{array}\right\} .
$$

Consequently, we get

$$
\Psi\left(\mathcal{T}^{q} w_{0}, \mathcal{T} u\right) \leq \max \left\{\begin{array}{l}
\eta\left(\Psi, \mathcal{T}, w_{0}\right)^{h k^{q}}, \eta\left(\Psi, \mathcal{T}, w_{0}\right)^{k^{q}}, \\
\Psi(u, \mathcal{T} u)^{k}, \Psi\left(w_{0}, \mathcal{T} u\right)^{k^{q}}
\end{array}\right\},
$$

for all $q \in \mathrm{N}$. Therefore, we obtain

$$
\lim \sup _{q \rightarrow \infty} \Psi\left(\mathcal{T}^{q} w_{0}, \mathcal{T} u\right) \leq \Psi(u, \mathcal{T} u)^{k} .
$$

Since $\Psi\left(w_{0}, \mathcal{T} u\right)<\infty$ and $\eta\left(\Psi, \mathcal{T}, w_{0}\right)<\infty$. Using the property $\left(\Psi_{3}\right)$, we get

$$
\Psi(\mathcal{T} u, u) \leq \lim \sup \Psi\left(\mathcal{T}^{q} w_{0}, \mathcal{T} u\right)^{h} \leq \Psi(u, \mathcal{T} u)^{h k} .
$$

which implies that

$$
\Psi(\mathcal{T} u, u)=1
$$

Since $\Psi(u, \mathcal{T} u)<\infty$ and $h k<1$. Then $u$ is the fixed point of $\mathcal{T}$. By using Proposition 3.2, we have

$$
\Psi(u, u)=1 \text {. }
$$

For uniqueness, suppose that $u^{\prime} \in \mathcal{X}$ is another fixed point of $\mathcal{T}$ such that $\Psi\left(u, u^{\prime}\right)<\infty$ and $\Psi\left(u^{\prime}, u^{\prime}\right)<\infty$. By using Proposition 3.2, we have

$$
\Psi\left(u^{\prime}, u^{\prime}\right)=1
$$

Since $\mathcal{T}$ is a Js k-quasicontraction, we obtain

$$
\Psi\left(u, u^{\prime}\right)=\Psi\left(\mathcal{T} u, \mathcal{T} u^{\prime}\right) \leq \Psi(u, u)^{k} .
$$

This implies that

$$
u=u^{\prime} \text {. }
$$

Corollary 3.4 Let $\left(\mathcal{X}, d_{m}\right)$ be a complete dislocated multiplicative metric space and let $\mathcal{T}: \mathcal{X} \rightarrow \mathcal{X}$ a mapping for which there exists $\lambda \in(0,1)$, such that

$$
d_{m}(\mathcal{T} w, \mathcal{T} y) \leq\left[J_{m}(w, y)\right]^{\lambda}, \text { for all } w, y \in \mathcal{X},
$$

where

$$
J_{m}(w, y)=\max \left\{\begin{array}{l}
d_{m}(w, y), d_{m}(w, T(w)), d_{m}(y, T(y)), \\
d_{m}(w, T(y)), d_{m}(y, T(w))
\end{array}\right\},
$$

if there exists $w_{0} \in \mathcal{X}$ such that

$$
\sup \left\{d_{m}\left(\mathcal{T}^{i}\left(w_{0}\right), \mathcal{T}^{j}\left(w_{0}\right)\right): i, j \in \mathrm{N}_{0}\right\}<\infty .
$$

Then $\mathcal{T}$ has a unique fixed point. Moreover, the sequence $\left\{\mathcal{T}^{q}\left(w_{0}\right)\right\}$ converges to fixed point of $\mathcal{T}$.

Corollary 3.5 Let $(\mathcal{X}, \mathcal{B})$ be a complete multiplicative b-metric space with constant $s \geq 1$ and let $\mathcal{T}: \mathcal{X} \rightarrow \mathcal{X}$ be 
a mapping for which there exists $\lambda \in(0,1)$ with $\lambda s<1$, such that

$$
\mathcal{B}(\mathcal{T} x, \mathcal{T} y) \leq \mathcal{B}(x, y)^{\lambda}, \text { for all } x, y \in \mathcal{X},
$$

if there exist $x_{0} \in \mathcal{X}$ we have

$$
\sup \left\{\mathcal{B}\left(\mathcal{T}^{i}\left(x_{0}\right), \mathcal{T}^{j}\left(x_{0}\right)\right): i, j \in \mathrm{N}_{0}\right\}<\infty .
$$

Then $\mathcal{T}$ has a unique fixed point. Moreover, the sequence $\left\{\mathcal{T}^{q}\left(x_{0}\right)\right\}$ converges to fixed point of $\mathcal{T}$.

Example 3.6 Let $\mathcal{X}=[0,1]$ and $\Psi(x, y)=\alpha^{x+y}$, where $\alpha>1$ (a finite fixed real number). Clearly $\Psi$ is Js multiplicative metric space for all $x, y \in \mathcal{X}$. Let us define a function $\mathcal{T}: \mathcal{X} \rightarrow \mathcal{X}$ by

$$
\mathcal{T}(x)=\frac{x}{2}, 0 \leq x \leq 1 .
$$

Thus, with $k=\frac{1}{2}$

$$
\Psi\left(\mathcal{T}_{x}, \mathcal{T} y\right) \leq[J(x, y)]^{k} .
$$

Let $x_{0}=1$, then it is clear that $\eta\left(\Psi, \mathcal{T}, x_{0}\right)<\infty$. Now $\mathcal{T}_{x_{0}}=\frac{1}{2}, \mathcal{T}^{2} x_{0}=\frac{1}{2^{2}}, \mathcal{T}^{3} x_{0}=\frac{1}{2^{3}} \ldots \ldots . . . \mathcal{T}^{q} x_{0}=\frac{1}{2^{q}} \ldots$ and so on. Clearly $\mathcal{T}^{q} x_{0}$ is Cauchy sequence and all the properties of Theorem 3.3 are satisfied. So $\mathcal{T}$ has a unique fixed point.

\section{Results in a Js Multiplicative Metric Space with Partial Order}

Definition 4.1 Let $(\mathcal{X}, \Psi)$ be a Js multiplicative metric space with partial order $\preceq$ and let $\mathcal{T}: \mathcal{X} \rightarrow \mathcal{X}$ a mapping. Then $\mathcal{T}$ is weakly continuous if $x_{n} \subset \mathcal{X}$ converges to $x \in \mathcal{X}$, then there exists a subsequence $\left\{x_{n q}\right\}$ of $x_{n}$ such that $\left\{\mathcal{T}\left(x_{n q}\right)\right\}$ is convergent to $\mathcal{T}(x)$ as $(q \rightarrow \infty)$.

Definition 4.2 Let $\mathcal{X} \neq \varphi$ with partial order $\preceq$. A mapping $\mathcal{T}: \mathcal{X} \rightarrow \mathcal{X}$ is called nondecreasing if

$$
x \preceq y \Rightarrow \mathcal{T}(x) \preceq \mathcal{T}(y) \text { where } x, y \in \mathcal{X} .
$$

Definition 4.3 The pair $(\mathcal{X}, \preceq)$ is called regular if for every sequence $\left\{x_{n}\right\} \subset \mathcal{X}$ satisfies $x_{n} \preceq x_{n+1}$ for each $n \in \mathbb{N}$ with $\left\{x_{n}\right\}$ being convergent to $x \in \mathcal{X}$ then there exist a subsequence $\left\{x_{n q}\right\}$ of $\left\{x_{n}\right\}$ such that $x_{n q} \preceq x$ for every $q \in \mathbb{N}$.

Definition 4.4 A function $\mathcal{T}: \mathcal{X} \rightarrow \mathcal{X}$ is called weakly Js k-contraction for some $k \in(0,1)$ if $x \preceq y$ or $y \preceq x$ we have

$$
\Psi\left(\mathcal{T}_{x}, \mathcal{T} y\right) \leq \Psi(x, y)^{k}
$$

Theorem 4.5 Let $(\mathcal{X}, \Psi)$ be a complete Js multiplicative metric space with partial order $\preceq$ and let $\mathcal{T}: \mathcal{X} \rightarrow \mathcal{X}$ be a function. Assume that the following conditions satisfied:

(i) $\mathcal{T}$ be a weakly continuous;

(ii) $\mathcal{T}$ be a weakly Js k-contraction for some $k \in(0,1)$;

(iii) there exists $w_{0} \in \mathcal{X}$ such that $\eta\left(\Psi, \mathcal{T}, w_{0}\right)<\infty$ and $w_{0} \preceq \mathcal{T}\left(w_{0}\right)$;

(iv) $\mathcal{T}$ is nondecreasing.

Then, $\left\{\mathcal{T}^{n}\left(w_{0}\right)\right\}$ converges to $u \in \mathcal{X}$ such that $u$ is fixed point of $\mathcal{T}$. Moreover, if $\Psi(u, u)<\infty$, then $\Psi(u, u)=1$.

Proof. Since $\mathcal{T}$ is nondecreasing and $w_{0} \preceq \mathcal{T}\left(w_{0}\right)$ then for all $n \in \mathbb{N}_{0}$, we obtain

$$
\mathcal{T}^{n}\left(w_{0}\right) \preceq \mathcal{T}^{n+1}\left(w_{0}\right) .
$$

By transitivity of $\preceq$, for every $r, s \in \mathbb{N}_{0}$, we have

$$
r \leq s \Rightarrow \mathcal{T}^{r}\left(w_{0}\right) \preceq \mathcal{T}^{s}\left(w_{0}\right) .
$$

There, for each $n \in \mathbb{N}_{0}(n \geq 1), i, j \in \mathbb{N}_{0}, \quad \mathcal{T}^{n+i}\left(w_{0}\right)$ and $\mathcal{T}^{n+j}\left(w_{0}\right)$ are always comparable. As $\mathcal{T}$ is weak Js k-contraction for each $n \in \mathbb{N}, i, j \in \mathbb{N}_{0}$, we have

$$
\Psi\left(\mathcal{T}^{n+i} w_{0}, \mathcal{T}^{n+j} w_{0}\right) \leq \Psi\left(\mathcal{T}^{n-1+i} w_{0}, \mathcal{T}^{n-1+j} w_{0}\right)^{k} .
$$

This implies that

$$
\begin{aligned}
\eta\left(\Psi, \mathcal{T}, \mathcal{T}^{n} w_{0}\right) & \leq \eta\left(\Psi, \mathcal{T}, \mathcal{T}^{n-1} w_{0}\right)^{k} \\
& \leq \eta\left(\Psi, \mathcal{T}, \mathcal{T}^{n-2} w_{0}\right)^{k^{2}} .
\end{aligned}
$$

So, we obtain that

$$
\eta\left(\Psi, \mathcal{T}, \mathcal{T}^{n} w_{0}\right) \leq \eta\left(\Psi, \mathcal{T}, w_{0}\right)^{k^{n}} .
$$

Taking (5) into account and by the definition of $\eta$, for every $m, n \in \mathbb{N}_{0}$, we obtain

$$
\begin{aligned}
\Psi\left(\mathcal{T}^{n} w_{0}, \mathcal{T}^{n+m} w_{0}\right) & \leq \eta\left(\Psi, \mathcal{T}, \mathcal{T}^{n} w_{0}\right) \\
& \leq \eta\left(\Psi, \mathcal{T}, w_{0}\right)^{k^{n}} .
\end{aligned}
$$

Using the fact that $k \in(0,1)$ and $\eta(\Psi, \mathcal{T}, w)<\infty$, we get

$$
\lim _{n, m \rightarrow \infty} \Psi\left(\mathcal{T}^{n} w_{0}, \mathcal{T}^{n+m} w_{0}\right)=1
$$

This shows that $\mathcal{T}^{n}\left(w_{0}\right)$ is a Cauchy sequence. By completeness of $(\mathcal{X}, \Psi)$, we must have some $u \in \mathcal{X}$ such that $\mathcal{T}^{n}\left(w_{0}\right)$ is convergent to $u$. Since $\mathcal{T}$ is weakly continuous so there is a subsequence $\left\{\mathcal{T}^{n q}\left(w_{0}\right)\right\}$ of $\left\{\mathcal{T}^{n}\left(w_{0}\right)\right\}$ such that $\left\{\mathcal{T}^{n q+1}\left(w_{0}\right)\right\}$ is convergent to $\mathcal{T}(u)$ 
as $(q \rightarrow \infty)$. By uniqueness of the limit, we get $u=\mathcal{T}(u)$ and $u$ is a fixed point of $\mathcal{T}$. Now if $\Psi(u, u)<\infty$ then as $u \preceq u$ and $\mathcal{T}$ is weak js k-contraction, we have

$$
\Psi(u, u)=\Psi(\mathcal{T} u, \mathcal{T} u) \leq \Psi(u, u)^{k},
$$

which is possible only if

$$
\Psi(u, u)=1 .
$$

The weak continuity assumption of $\mathcal{T}$ in the previous theorem can be replaced by Definition 4.3 to obtain the following result.

Theorem 4.6 Let $(\mathcal{X}, \Psi)$ be a complete Js multiplicative metric space with partial order $\preceq$ and let $\mathcal{T}: \mathcal{X} \rightarrow \mathcal{X}$ be a function. Assume that the following conditions satisfied:

(i) $(\mathcal{X}, \preceq)$ is regular;

(ii) $\mathcal{T}$ be a weakly Js k-contraction for some $k \in(0,1)$;

(iii) there exists $w_{0} \in \mathcal{X}$ such that $\eta\left(\Psi, \mathcal{T}, w_{0}\right)<\infty$ and $w_{0} \preceq \mathcal{T}\left(w_{0}\right)$;

(iv) $\mathcal{T}$ is nondecreasing.

Then $\left\{\mathcal{T}^{n}\left(w_{0}\right)\right\}$ converges to $u \in \mathcal{X}$ such that $u$ is fixed point of $\mathcal{T}$. Moreover, if $\Psi(u, u)<\infty$, then $\Psi(u, u)=1$.

Proof. As we have proved in previous proof that $\mathcal{T}^{n}\left(w_{0}\right)$ is convergent to $u$. Moreover, we have

$$
\mathcal{T}^{n}\left(w_{0}\right) \preceq \mathcal{T}^{n+1}\left(w_{0}\right) \text { for all } n \in \mathbb{N}_{0} \text {. }
$$

Since $(\mathcal{X}, \preceq)$ is regular, there exists a subsequence $\left\{\mathcal{T}^{n q}\left(w_{0}\right)\right\}$ of $\left\{\mathcal{T}^{n}\left(w_{0}\right)\right\}$ such that $\mathcal{T}^{n q} \preceq u$ for each $q \in \mathbb{N}$. As $\mathcal{T}$ is weak Js k-contraction, we have

$$
\Psi\left(\mathcal{T}^{n q+1} w_{0}, \mathcal{T} u\right) \leq \Psi\left(\mathcal{T}^{n q} w_{0}, u\right)^{k} .
$$

Using the inequality above, we get

$$
\lim _{q \rightarrow \infty} \Psi\left(\mathcal{T}^{n q+1} w_{0}, \mathcal{T} u\right)=1
$$

This implies that $\left\{\mathcal{T}^{n q+1}\left(w_{0}\right)\right\}$ is converges to $\mathcal{T}(u)$. By uniqueness of the limit, we obtain

$$
\mathcal{T}(u)=u \text {. }
$$

As in the previous proof

$$
\Psi(u, u)<\infty \Rightarrow \Psi(u, u)=1 .
$$

\section{Competing Interests}

The authors declare that they have no competing interests.

\section{References}

[1] M. Ozavsar and A. C. Cervikel, Fixed Points of Multiplicative Contraction Mappings on Multiplicative Metric Spaces, Journal of Engineering Technology and Applied Sciences, 2(2), 2017, 65-79.

[2] C. Mongkolkeha, and W. Sintunavarat, Best Proximity Points for Multiplicative Proximal Contraction Mapping on Multiplicative Metric Spaces, J. Nonlinear Sci. Appl, 8(6); 2015, 1134-1140:

[3] M. Abbas, B. Ali, and YI. Suleiman, Common Fixed Points of Locally Contractive Mappings in Multiplicative Metric Spaces with Application, International Journal Of Mathematics and Mathematical Sciences, 2015; 2015, 1-7.

[4] M. Abbas, M. D. Sen, and T. Nazir, Common Fixed Points of Generalized Rational Type Cocyclic Mapping in Multiplicative Metric Spaces, Discrete Dynamics in Nature and Society, 2015; 2015, 1-10.

[5] A. E. Al-Mazrooei, D. Lateef, and J. Ahmad, Common Fixed Point Theorems for Generalized Contractions, Journal Of Mathematical Analysis, 8(3); 2017; 157-166.

[6] C. Mongkolkeha, and W. Sintunaravat, Optimal Approximate Solutions for Multiplicative Proximal Contraction Mappings in Multiplicative Metric Spaces, Proceedings of National Academy of Sciences, 86(1); 2016, 15-20.

[7] E. Ameer and M. Arshad, Two new generalizations for F-contraction on closed ball and fixed point theorems with application, J. Mathematical Extension, 11, 2017, 43-67.

[8] M. U. Ali, T. Kamran, and A. Kurdi, Fixed Point Theorems in b-multiplicative Metric Spaces, U. P. B. Sci. Bull., Series A, 79(3) 2017, 107-116.

[9] B. Zada and U. Riaz, Some Fixed point Results on multiplicative b-metric like spaces, Turkish Journal of Analysis and Number Theory, 4(5), 118-131 (2016).

[10] M. Jleli, B. Samet, A generalized metric space and related fixed point theorems, Fixed point theory Appl., 2015: 61, 2015.

[11] I. Altun, N. Al Arifi, M. Jleli, A. Lashin, and B. Samet, Feng-Liu type fixed point results for multivalued mappings on $\{$ JS $\}$-metric spaces, Journal of Nonlinear Sciences and Appl., 9(6), 2016.

[12] E. Karapınar, D. O'Regan, A. F. R. L. Hierro, N. Shahzad, Fixed point theorems in new generalized metric spaces, J. Fixed Point Theory Appl. 18 (2016), 645-671.

[13] M. Noorwali, H. H. Alsulami, E. Karapinar, Some extensions of fixed point results over quasi-JS-spaces, J. Funct. Spaces. (2016), 2016: 6963041. 